\title{
Size Dependence In The Disordered Kondo Problem
}

\author{
Ivar Martin, Yi Wan, and Philip Phillips \\ Loomis Laboratory of Physics \\ University of Illinois at Urbana-Champaign \\ 1100 W.Green St., Urbana, IL, 61801-3080
}

\begin{abstract}
We study here the role randomly-placed non-magnetic scatterers play on the Kondo effect. We show that spin relaxation effects (with time $\tau_{s}^{o}$ ) in the vertex corrections to the Kondo self-energy lead to an exact cancellation of the singular temperature dependence arising from the diffusion poles. For a thin film of thickness $L$ and a mean-free path $\ell$, disorder provides a correction to the Kondo resistivity of the form $\tau_{s}^{o} /\left(k_{F} L \ell^{2}\right) \ln T$ that explains both the disorder and sample-size depression of the Kondo effect observed by Blachly and Giordano (PRB 51, 12537 (1995)).
\end{abstract}

PACS numbers:72.10.Fk, 72.15.Nj, 75.20.Hr

Typeset using REVTEX 
At low temperatures, the resistivity of a metal alloy acquires a logarithmic temperature dependence [1] in response to spin-flip scattering between local magnetic impurities and the conduction electrons. This behaviour persists down to a temperature (the Kondo temperature, $T_{k}$ ) where the magnetic impurities and conduction electrons begin to condense into singlet states. While perturbation theory is sufficient to establish the existence of the $\ln T$ term, its presence ultimately signifies that perturbation theory is breaking down. Alternatively, spin-flip scattering between conduction electrons and localized magnetic centers has a singular frequency $(\omega)$ dependence. Magnetic impurities are not alone in this respect. It is well-known that even non-magnetic impurities can generate a singular $(\ln \omega$ in $d=2)$ frequency dependence in the conductivity [2]. In a sample containing both magnetic and non-magnetic impurities, the question arises: which singularity ultimately wins or can the interplay between the singularities lead to a suppression of either localization or the Kondo effect? In this letter, we resolve these questions.

The motivation for this study is two-fold. First, while there have been numerous treatments of this problem [4]- [7], a clear consensus has not been reached. To illustrate, Everts and Keller [4] were first to show that non-magnetic scattering contributes a $1 / \sqrt{T}$ to the Kondo self-energy that dominates the Kondo $\ln T$ singularity at low temperatures in $\mathrm{d}=3$. Bohnen and Fisher [5] argued, however, that such a term would not survive in the conductivity. More recently, Ohkawa, Fukuyama, and Yosida [6] showed that disorder results in a singularity of the form $T^{d / 2-2}$ in the conductivity. At low temperatures this singularity dominates the Kondo $\ln T$. As a result, these groups conclude that static disorder can mask the Kondo resistivity as $T \rightarrow 0$. On the experimental side, Blachly and Giordano [ 8 ] recently measured the conductivity in a series of thin films containing magnetic as well as non-magnetic impurities. They found no evidence for the $T^{d / 2-2}$ singularity but observed instead a suppression of the Kondo resistivity as the strength of the disorder increased. Earlier experiments by Korn [9] also failed to observe the $T^{d / 2-2}$ singularity but observed instead an enhancement in the Kondo resistivity. The point of agreement between these experiments is that disorder couples non-trivially to the Kondo effect and ultimately modi- 
fies the coefficient of the $\ln T$ dependence. Given the strong dimensional dependence of the localization transition, disorder could eventually lead to a sample size dependence of the Kondo effect.

At the outset, we set aside the still controversial issue (ref. 8c) of the sample size dependence and focus on the seemingly straightforward problem of the role non-magnetic disorder plays in the Kondo effect. The new wrinkle we introduce in this problem is the feedback effect spin scattering has on localization. While it is standard to consider the direct influence of localization on the Kondo effect, the reverse effect has not been included [10]. Nonetheless, it is well-known that electron scattering by disordered Heisenberg spins introduces a cutoff of the diffusion pole in both the particle-hole (diffuson) and particleparticle (Cooperon) channels except for the $S=0$ particle-hole channel [11]. When spinscattering is included in the diffusion propagators, the fate of the $T^{d / 2-2}$ singularity rests on whether the $S=0$ particle-hole propagator contributes to the Kondo self-energy. We show explicitly it does not.

The starting point for our analysis is a model Hamiltonian $H=H_{o}+H_{s d}$ that contains both normal impurities

$$
H_{o}=\sum_{k \sigma}\left(\varepsilon_{k}-\varepsilon_{F}\right) a_{k \sigma}^{\dagger} a_{k \sigma}+\frac{v}{\Omega} \sum_{k, k^{\prime}, i} e^{\mathbf{i}\left(\mathbf{k}-\mathbf{k}^{\prime}\right) \cdot \mathbf{R}_{\mathbf{i}}} a_{k \sigma}^{\dagger} a_{k^{\prime} \sigma}
$$

as well as magnetic scatterers

$$
H_{s d}=-\frac{J}{\Omega} \sum_{R_{n}, k, k^{\prime}, \sigma, \sigma^{\prime}} e^{\mathbf{i}\left(\mathbf{k}-\mathbf{k}^{\prime}\right) \cdot \mathbf{R}_{\mathbf{n}}} \sigma_{\sigma, \sigma^{\prime}} \cdot \mathbf{S}_{\mathbf{n}} a_{k \sigma}^{\dagger} a_{k^{\prime} \sigma^{\prime}} .
$$

The operator $a_{k \sigma}^{\dagger}$ creates an electron in a plane wave state with momentum $k$ and spin $\sigma$ and energy $\varepsilon_{k}, v$ measures the strength of the scattering with the non-magnetic disorder, $J$ is the exchange interaction, $R_{n}$ denotes the position of the impurities, magnetic or otherwise, $\mathbf{S}_{n}$ is the spin operator for the magnetic impurity at site $n, \sigma$ is the Pauli spin operator, and $\Omega$ is the volume. The two natural timescales in this problem are, $\tau_{s}^{o}$ and $\tau_{o}$, the magnetic and non-magnetic scattering times. In terms of the density of states of the host metal, $\rho_{o}$ and the concentrations of magnetic and non-magnetic scatterers, $n_{s}$ and $n_{o}$, respectively, 
we have that $\hbar / 2 \tau_{s}^{o}=3 \pi n_{s} \rho_{o}|J|^{2} / 4$ and $\hbar / 2 \tau_{o}=\pi n_{o} \rho_{o}|v|^{2}$. The total scattering rate is $1 / \tau=1 / \tau_{s}^{o}+1 / \tau_{o}$. To measure the strength of the non-magnetic disorder, we define $\lambda=\hbar /\left(2 \pi \varepsilon_{F} \tau_{o}\right)$. We assume that the concentration of localized spins is dilute so that longrange spin glass effects are irrelevant. Also, we work in the regime in which normal impurity scattering dominates, $1 / \tau_{o} \gg 1 / \tau_{s}^{o}$

To evaluate the conductivity above $T_{k}$, we must first calculate the Kondo self energy. To include the dynamical effects of the localized spins, it is sufficient to calculate the self energy to third order in the exchange interaction $J$. At this order, static disorder can be included by decorating the single and double spin-flip vertices with Cooperon and diffuson propagators [6], [7]. In previous work [6], [7], spin-independent Cooperons and diffusons of the form $C(Q, \omega)=D(Q, \omega) \propto \frac{1}{\left(D Q^{2}-i \omega\right)}$ were used where $Q$ and $\omega$ are the net momentum and energy transfer and $D=2 \hbar \varepsilon_{F} \tau / d m$ is the diffusion constant. However, this is inconsistent because $C(Q, \omega)$ and $D(Q, \omega)$ are coupled to electron lines of different spin. Such propagators are well-known [11] to depend on spin and hence we include explicitly the spin dependence here. If all scattering processes are treated in the first Born approximation, the Cooperon propagator [1] is transformed to

$$
\begin{aligned}
C_{\alpha \beta \gamma \delta}= & \frac{\hbar^{2}}{8 \pi \rho_{o} \tau^{2}\left(D Q^{2}-i \omega+2 / \tau_{s}^{o}\right)}\left(\delta_{\alpha \beta} \delta_{\gamma \delta}-\sigma_{\alpha \beta} \cdot \sigma_{\gamma \delta}\right) \\
& +\frac{\hbar^{2}}{8 \pi \rho_{o} \tau^{2}\left(D Q^{2}-i \omega+2 / 3 \tau_{s}^{o}\right)}\left(3 \delta_{\alpha \beta} \delta_{\gamma \delta}+\sigma_{\alpha \beta} \cdot \sigma_{\gamma \delta}\right)
\end{aligned}
$$

and the diffuson becomes

$$
\begin{aligned}
D_{\alpha \beta \gamma \delta}= & \frac{\hbar^{2}}{8 \pi \rho_{o} \tau^{2}\left(D Q^{2}-i \omega\right)}\left(\delta_{\alpha \beta} \delta_{\gamma \delta}+\sigma_{\alpha \beta} \cdot \sigma_{\gamma \delta}\right) \\
& +\frac{\hbar^{2}}{8 \pi \rho_{o} \tau^{2}\left(D Q^{2}-i \omega+4 / 3 \tau_{s}^{o}\right)}\left(3 \delta_{\alpha \beta} \delta_{\gamma \delta}-\sigma_{\alpha \beta} \cdot \sigma_{\gamma \delta}\right) .
\end{aligned}
$$

The survival of the diffusion pole in the spin- dependent diffuson is a consequence of particlehole conservation. The two terms in each of these propagators correspond to singlet and triplet scattering, respectively. Pairs of spin indices $\alpha \beta$ and $\gamma \delta$ are indexed chronologically along the particle lines that comprise the diffusion ladder diagrams. 
The diagrams shown in Fig. 1a contain the dominant quantum corrections to the Kondo self-energy at third order in the presence of disorder. The sum of all such diagrams is

$$
\begin{aligned}
\Sigma_{3 q}\left(k, i \epsilon_{n}\right)= & \frac{2}{\beta^{2}} \sum_{\omega_{\ell}, \omega_{m}, Q, q, \pm} V_{\alpha \beta \nu \eta}^{ \pm}\left(i \omega_{\ell}, i \omega_{m}\right) G\left(i \epsilon_{n}+i \omega_{m}, q\right) \times \\
& {\left[G\left(i \epsilon_{n}+i \omega_{\ell}, k+Q\right)+n_{o}|v|^{2} \sum_{k^{\prime}} G^{2}\left(i \epsilon_{n}, k^{\prime}\right) G\left(i \epsilon_{n}+i \omega_{\ell}, k^{\prime}+Q\right)\right] \times } \\
& \left(D_{\sigma \alpha \beta \gamma}\left(i \omega_{\ell}, Q\right) D_{\gamma \nu \eta \sigma}\left(i \omega_{\ell}, Q\right)+C_{\sigma \alpha \gamma \nu}\left(i \omega_{\ell}, Q\right) C_{\beta \gamma \eta \sigma}\left(i \omega_{\ell}, Q\right)\right)
\end{aligned}
$$

where $G(i \epsilon, q)$ is the electron Green function

$$
G(i \epsilon, q)=\frac{1}{i \epsilon+\epsilon_{F}-\hbar^{2} q^{2} / 2 m+i(\hbar / 2 \tau) \operatorname{sgn}(\epsilon)},
$$

the electron energies are the Matsubara frequencies, $\epsilon_{n}=(2 n+1) \pi T$, the psuedofermion energies are $z_{k}=(2 k+1) \pi T, \omega_{\ell}=2 l \pi T, D Q^{2}<\hbar / \tau_{o}$ and $\left(\epsilon_{n}+\omega_{\ell}\right) \omega_{\ell}<0$. We have set $k_{B}=1$. The factor of 2 arises from the two possible couplings of the diffusion progagators to the internal electron lines and the \pm from the two orientations of the psuedofermion loops. The psuedofermion part involves a trace over the components of the impurity spin operators and hence simplifies to

$$
V_{\alpha \beta \nu \eta}^{ \pm}\left(i \omega_{l}, i \omega_{m}\right)=\frac{1}{4} J^{3} n_{s} \beta\left[\frac{1}{i \omega_{\ell}}\left(\delta_{m 0}-\delta_{\ell m}\right)\left(1-\delta_{\ell 0}\right)+\frac{1}{i \omega_{m}} \delta_{\ell 0}\left(1-\delta_{m 0}\right) \pm \frac{\beta}{2} \delta_{m 0} \delta_{\ell 0}\right]\left(\sigma_{\alpha \beta}^{a} \sigma_{\nu \eta}^{a}\right)
$$

From the psuedofermion contribution, we see that the sum over the spin indices separates into two identical sums of the form, $\sum_{\alpha \beta} D_{\sigma \alpha \beta \gamma} \sigma_{\alpha \beta}^{a}$. If we use the identity $\sum_{\alpha \beta}\left(\sigma_{\nu \alpha} \cdot \sigma_{\beta \gamma}\right) \cdot \sigma_{\alpha \beta}^{a}=$ $-\sigma_{\nu \gamma}^{a}$, we find immediately that the cancellation of the divergent diffusion terms

$$
\sum_{\alpha \beta} D_{\nu \alpha \beta \gamma}^{S=0} \sigma_{\alpha \beta}^{a} \propto \sum_{\alpha \beta}\left(\delta_{\nu \alpha} \delta_{\beta \gamma}+\sigma_{\nu \alpha} \cdot \sigma_{\beta \gamma}\right) \sigma_{\alpha \beta}^{a}=0
$$

from the $3^{\text {rd }}$ order Kondo self-energy is exact. To any order in $J$, the cancellation of the diffusion pole can be seen as follows. In the most divergent approximation, each diffuson encircles a vertex that is exactly equal to the Abrikosov 12 vertex function $\Gamma \propto \sigma \cdot \mathbf{S}$. When this function is now multiplied by $D^{S=0}$ and summed over the spin indices, the cancellation to all orders follows immediately from Eq. (8). This is one of the principal results of this 
paper. The cancellation of the $S=0$ component of the diffuson is fundamentally tied to the fact that the Kondo interaction does not conserve spin. Further, it signifies that the resultant conductivity is independent of the pure charge density propagator. Summing over the spin indices in the remaining propagators in the self energy reduces the problem to one in which the diffuson and Cooperon are spin independent: $\tilde{D}=\hbar^{2} /\left(2 \pi \rho_{o} \tau^{2}\right)\left(D Q^{2}-i \omega+4 / 3 \tau_{s}^{o}\right)^{-1}$ and $\tilde{C}=\hbar^{2} /\left(4 \pi \rho_{o} \tau^{2}\right)\left[\left(D Q^{2}-i \omega+2 / \tau_{s}^{o}\right)^{-1}+\left(D Q^{2}-i \omega+2 / 3 \tau_{s}^{o}\right)^{-1}\right]$. When $1 / \tau_{s}^{o}=0$ (or equivalently, $T \gg \hbar / \tau_{s}^{o}$ ), we recover the standard form for these propagators.

To calculate the resistivity, we evaluate the standard self-energy as well as the Cooperon weak-localization diagrams [6]. Because the results of the calculation are rather lengthy, we present here only the asymptotic behaviour. In the limit $T \gg \hbar / \tau_{s}^{o}$ (as in the case when the magnetic impurities are dilute), we recover the inverse temperature dependence

$$
\frac{\hbar}{2 \tau^{C}}=\frac{\hbar}{2 \tau^{D}}=\frac{-\pi \hbar \rho_{o} \lambda J}{3 \tau_{o}} \frac{\hbar}{\tau_{s}^{o} T} \ll-\rho_{o} \lambda J \frac{\hbar}{\tau_{o}}
$$

of refs. ( [7], [6]). In the opposite regime, $T \ll \hbar / \tau_{s}^{o}$, the limiting forms of the Cooperon and diffuson relaxation times

$$
\frac{\hbar}{2 \tau^{D}}+\frac{\hbar}{2 \tau^{C}}=-\left(\frac{5}{2}+\frac{3 \ln 3}{4}\right) \rho_{o} \lambda J \frac{\hbar}{\tau_{o}} \ln \frac{\hbar}{T \tau_{s}^{o}}
$$

are both logarithmic functions of temperature.

The final contribution to the relaxation time comes from the the Cooperon weaklocalization diagram. In two dimensions in the presence of spin-flip scattering, the weaklocalization contribution is $\Delta \sigma_{l o c}=-e^{2} /\left(2 \pi^{2} \hbar\right) \ln \left(\sqrt{3} \tau_{\epsilon s} / \tau_{o}\right)$, where $\hbar / 2 \tau_{\epsilon s}=8 \hbar /\left(3 \tau_{s}^{o}\right)(1-$ $\left.\rho_{o} J \ln \left(\epsilon_{F} / T\right)\right)$. Physically, $\tau_{\epsilon s}$ plays the role of the inelastic scattering time in the weaklocalization correction. Inclusion of the $3^{\text {rd }}$ order correction to the spin scattering time, enhances the spin-flip scattering rate, thereby weakening the effects of localization. To see this more clearly, we expand the argument of the logarithm for temperatures well above the Kondo temperature:

$$
\Delta \sigma_{l o c}=\frac{-e^{2}}{2 \pi^{2} \hbar} \ln \left(\frac{3 \sqrt{3} \tau_{s}^{o}}{8 \tau_{o}}\right)-\frac{e^{2}}{2 \pi^{2} \hbar} \rho_{o} J \ln \left(\frac{\epsilon_{F}}{T}\right)
$$


We see clearly that the Kondo interaction reduces the weak localization correction because $J<0$.

We collect all the contributions discussed above to determine the conductivity. In the temperature range $T_{k} \ll T<\hbar / \tau_{s}^{o}$, Cooperon, diffuson, and weak-localization corrections are logarithmic in temperature. Combining the results from Eq. (10) with the weaklocalization correction, we find that the magnitude of the logarithmic part of the conductivity

$$
\Delta \sigma^{T}=\sigma_{o} \frac{4 \tau_{o} \rho_{o} J}{\tau_{s}^{o}}\left(1+1.4 \lambda \frac{\tau_{s}^{o}}{\tau_{o}}\right) \ln \frac{\epsilon_{F}}{T}
$$

is enhanced by disorder. The first term in this expression arises from the unperturbed Kondo effect and the latter from the interplay with disorder. Inclusion of disorder in the self energy always enhances the Kondo resistivity by increasing repetitive scattering at magnetic impurities.

For temperatures $T \gg \hbar / \tau_{s}^{o}$, the self-energy contribution to the relaxation time scales as $1 / T$, whereas the weak-localization correction is proportional to $\ln T$. However, comparison of the magnitude of these corrections (see Eqs. (9) and (11)) reveals that the weak-localization term dominates and the magnitude of the resultant logarithmic correction

$$
\Delta \sigma^{T}=\sigma_{o} \frac{4 \tau_{o} \rho_{o} J}{\tau_{s}^{o}}\left(1-\frac{\lambda \tau_{s}^{o}}{4 \tau_{o}}\right) \ln \frac{\epsilon_{F}}{T}
$$

is suppressed by the disorder. The ratio $\lambda / \tau_{o}$ scales as $1 / \ell^{2}$, where $\ell$ is the mean-free path. We see then that in the dilute impurity regime, disorder suppresses the Kondo effect. The crossover from enhancement to suppression of the Kondo effect occurs because the magnitude and functional dependence of the quantum corrections to the self-energy are determined by the shortest of two length scales: the phase-breaking length, $L_{\phi}=\sqrt{D \tau_{s}^{o} / \hbar}$ and the diffusion length, $L_{T}=\sqrt{D / T}$. The latter arises because coupling of diffusion propagators to internal electron lines in the self-energy leads to an effective electron-electron interaction.

Let us now apply our results to the experiments on thin films by Blachly and Giordano 8]. In all of their samples the film thickness $L$ satisfied the inequality $\ell<L \ll L_{\phi}$. Hence, we can treat the films as quasi- $2 \mathrm{~d}$ with respect to localization, but because $\ell<L$ the electron 
gas is characterized by a 3-dimensional density of states $\rho_{o}=1 /(2 \pi)^{2}\left(2 m / \hbar^{2}\right)^{3 / 2} \epsilon^{1 / 2}$ with a diffusion constant given by $D=2 \hbar \epsilon_{F} \tau_{o} / 3 m$. The summation on $Q$ in the Cooperon and diffuson is restricted to small momentum transfers such that $D Q^{2}<1 / \tau_{o}$. However, for thicknesses of the sample on the order of $\ell$, the smallest wave vector in the transverse direction does not satisfy this constraint. To rectify this problem, Volkov [13 showed that surface boundary conditions must be treated consistently. For thin films, his treatment shows that the boundaries always give rise to a strictly 2-dimensional weak-localization correction and an explicit finite size dependence. To account for the former, the momentum integration in the Cooperon and diffuson must be restricted to the plane. The density of states that arises from converting the sum to an integral will be the 2-dimensional density of states $\rho_{o}^{2 D}=\pi \rho_{o} /\left(k_{F} L\right)$. Hence, the self-energy diagrams will generate a size-dependence to the conductivity. The explicit finite-size weak-localization correction is [13] $\Delta \sigma_{l o c}=$ $-e^{2} /\left(2 \pi^{2} \hbar L\right) \ln \left(\sqrt{3} \tau_{\epsilon s} / \tau_{o}(\sinh (L / \ell)(\ell / L))\right)$. The size-dependence in the logarithm yields an effective size dependence in the spin-relaxation time. However, this will not affect the temperature dependence of the conductivity. Hence, the only size dependence that is coupled to the temperature is the $1 / L$ prefactor of the weak-localization correction.

We now combine these results in the low and high-temperature limits discussed earlier. In the two limits, we obtain

$$
\Delta \sigma^{T}=\left\{\begin{array}{lll}
\sigma_{o} \frac{4 \tau_{o} \rho_{o} J}{\tau_{s}^{o}}\left(1+\frac{2.3 \hbar \tau_{s}^{o}}{\pi m k_{F} L \ell^{2}}\right) \ln \frac{\epsilon_{F}}{T} \text { if } & T_{k} \ll T<\hbar / \tau_{s}^{o} \\
\sigma_{o} \frac{4 \tau_{o} \rho_{o} J}{\tau_{s}^{o}}\left(1-\frac{1.2 \hbar \tau_{s}^{o}}{\pi m k_{F} L \ell^{2}}\right) \ln \frac{\epsilon_{F}}{T} \text { if } & T_{k}, \hbar / \tau_{s}^{o} \ll T
\end{array}\right.
$$

an explicit size and disorder correction that scales as $1 /\left(\ell^{2} L\right)$. In the concentrated impurity limit $T<\hbar / \tau_{s}^{o}$, increasing disorder enhances the resistivity. In $\mathrm{Cu}(\mathrm{Fe})$ alloys at impurity concentrations ranging from $0.3-2.1 \%$, Korn observed an enhancement in the Kondo resistivity that is consistent with the first equation above. However, in the dilute limit, $T \gg \hbar / \tau_{s}^{o}$, we predict a suppression of the Kondo effect as the disorder is increased and the size of the sample decreases. In the experiments of Blachly and Giordano [8], $\hbar / \tau_{s}^{o} \approx 0.1 K$ which is much less than the Kondo temperature for $C u(F e)$. The second of equations should 
be valid. Fig. 2 shows a comparison between the experimental data and the theoretical predictions. The best fit to the data was obtained with $\tau_{s}^{o}=0.52 n s$ which is consistent with the

experimental range of $10^{-10} \mathrm{~s}$. As is evident, theory and experiment are in good agreement. We also obtained quantitative agreement with the experimental data when the sample size was varied. We conclude that disorder can suppress the Kondo resistivity and give rise to a sample size dependence of the form $1 /\left(\ell^{2} L\right)$. We note in closing that a recent theory of the Kondo size dependence in clean samples has been proposed by Ujsaghy, Zawadowski, and Gyorffy [14]. This approach applies strictly in the ballistic case where $\ell \gg L$.

\section{ACKNOWLEDGMENTS}

We thank Dan Ralph for making us aware of the Giordano experiments and Nick Giordano, Yuri Lyanda-Geller and Eduardo Fradkin for useful discussions on spin-scattering. This work is supported in part by the NSF grants No. DMR94-96134. 


\section{REFERENCES}

[1] J. Kondo, Prog. Theor. Phys. 32, 37 (1964).

[2] E. Abrahams, et al., Phys. Rev. Lett. 42, 673 (1979).

[3] P. W. Anderson, et. al., Phys. Rev. Lett. 43, 718 (1979).

[4] H. U. Everts and Keller, Z. Phys. 240, 281 (1970).

[5] K.-P. Bohnen and K. H. Fischer, J. Low. Temp. Phys. 12, 559 (1970).

[6] F. J. Ohkawa, et. al., J. Phys. Soc. Jpn. 52, 1701 (1983); For a review see F. J. Ohkawa, Prog. Theor. Phys. Suppl. 84, 166 (1985).

[7] K. Vladar and G. T. Zimanyi, J. Phys. C 18, 3739 (1985); see also, S. Suga, et. al., J. Phys. Soc. Jpn. 55, 2515 (1986).

[8] M. A. Blachly and N. Giordano Phys. Rev. B 51, 12537 (1995); b) J. F. Di Tusa, et. al. Phys. Rev. Lett. 68, 678 (1992); c) V. Chandrasekhar, et. al. Phys. Rev. Lett. 72, $2053(1994)$.

[9] D. Korn, Z. Physik 238, 275 (1970).

[10] In ref. 8, spin relaxation is included in the Cooperon contribution to the conductivity. However, the equivalent contribution in the self-energy was ignored.

[11] B. L. Altshuler, et. al. Quantum Theory of Solids, Ed. I. M. Lifshits, (MIR, Moscow), 1982; D. Belitz and T. R. Kirkpatrick, Rev. Mod. Phys. 66, 261 (1994).

[12] A. A. Abrikosov, Physica 2, 5 (1965).

[13] V. A. Volkov, JETP Lett. 36, 475 (1982).

[14] O. Ujsaghy, et. al. Phys. Rev. Lett. 76, 2378 (1996). 


\section{FIGURES}

FIG. 1. Feynman diagrams contributing to the Kondo self-energy $(\Sigma)$. The dashed lines correspond to Abrikosov psuedofermions and the double solid lines to diffusons and double dashed lines to the Cooperons. The Greek letters indicate the spin. The $X$ indicates a single non-magnetic impurity scattering event.

FIG. 2. Comparison of the theroretical prediction for the Kondo resistivity predicted from the second of Eq. (14) with the experimental data of Blachly and Giordano ( [8]) Fig. 7. The horizontal axis measures the strength of the static disorder through the mean-free path. 

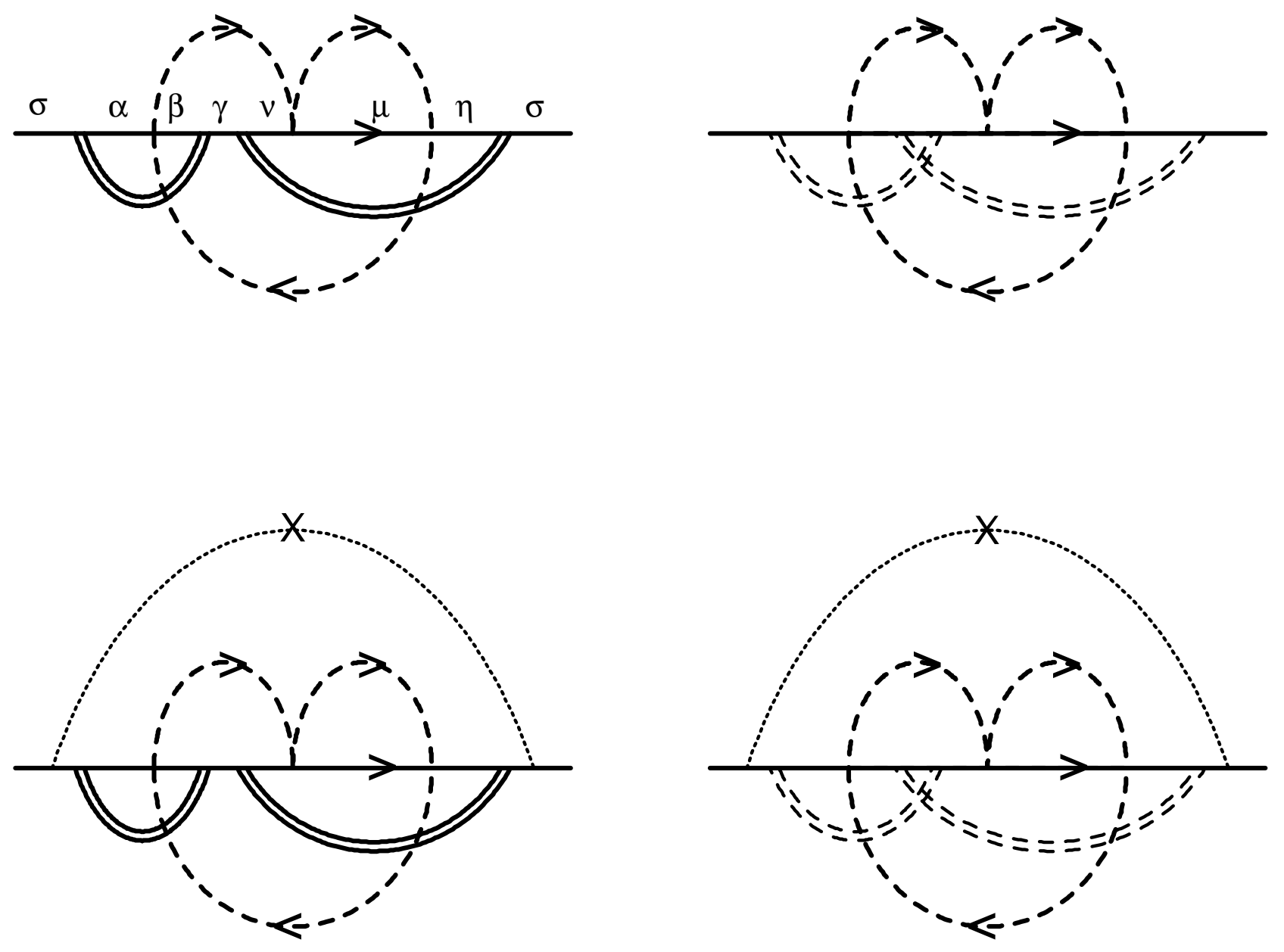

Fig. $1 a$. 


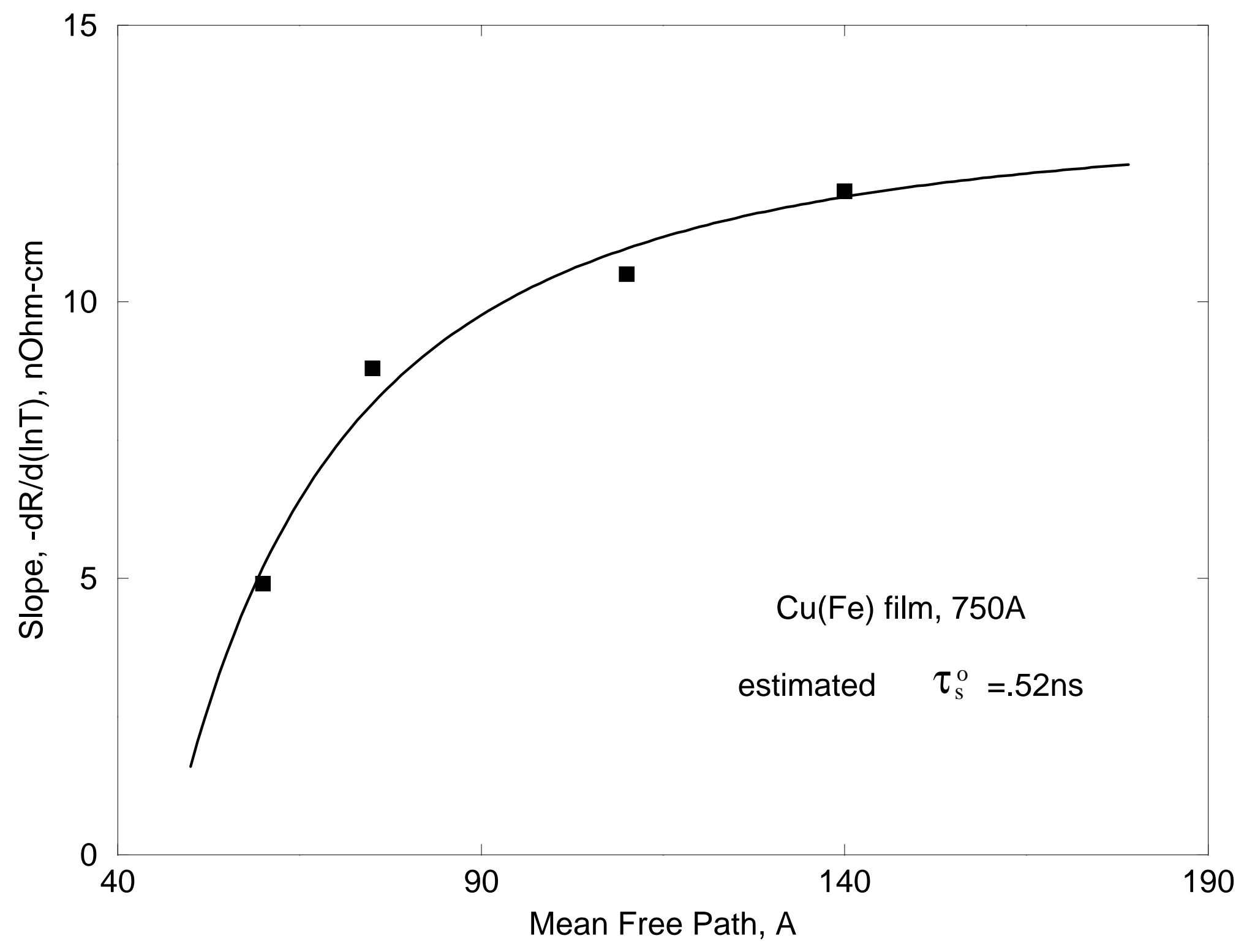

